\title{
ACCURATE EVALUATION OF EUROPEAN AND AMERICAN OPTIONS UNDER THE CGMY PROCESS*
}

\author{
ARIEL ALMENDRAL ${ }^{\dagger}$ AND CORNELIS W. OOSTERLEE ${ }^{\dagger}$
}

\begin{abstract}
A finite-difference method for integro-differential equations arising from Lévy driven asset processes in finance is discussed. The equations are discretized in space by the collocation method and in time by an explicit backward differentiation formula. The discretization is shown to be second-order accurate for a relevant parameter range determining the degree of the singularity in the Lévy measure. The singularity is dealt with by means of an integration by parts technique. An application of the fast Fourier transform gives the overall amount of work $O\left(N_{t} N \log N\right)$, rendering the method fast.
\end{abstract}

Key words. partial integro-differential equations, collocation method, option pricing

AMS subject classifications. 45K05, 45D05, 65R20, 91B28

DOI. $10.1137 / 050637613$

1. Introduction. In a seminal paper from 1973, Black and Scholes [8] derived a PDE for option prices, when asset prices behave according to the geometric Brownian motion. The pricing formulas obtained in that paper represented a major breakthrough in understanding financial derivatives, to such an extent that financial institutions and traders immediately adopted the new methodology. Later empirical studies revealed that the normality of the log-returns, as assumed by Black and Scholes, could not capture features like heavy tails and asymmetries observed in market-data logreturn densities [12]. The Black-Scholes model assumes in addition constant problem parameters, interest rate, and volatility for the time until the option's expiration (also called maturity). This contradicts the existence of the so-called volatility smile: A numerical inversion of the Black-Scholes formula based on data from different strike prices and fixed maturity resembles a skew or a smile, implying nonconstant volatility. This inconsistency is said to be one of the causes for famous market crashes.

To explain these empirical observations, a number of alternate models have appeared in the financial literature: stochastic volatility [25, 28], deterministic local volatility [17, 20], jump-diffusion [29, 33], and infinite activity Lévy models [7, 15, 21]. Each of these models has its advantages and disadvantages. Jump-diffusion and infinite activity Lévy models are attractive since they can capture the jump patterns exhibited by some stocks and they are more realistic when pricing options close to maturity [18]. Processes with infinite activity, without a diffusion component, represent a family that describes the high activity of the prices, while at the same time they reflect the empirical features desirable in a good model. The pricing equations are, however, numerically more challenging, and the market turns out to be incomplete in the sense that a strategy leading to an instantaneous risk-free portfolio does not exist in general $[16,34]$.

${ }^{*}$ Received by the editors August 5, 2005; accepted for publication (in revised form) July 6, 2006; published electronically January 12, 2007. This research was supported by the Dutch government through the national program BSIK: Knowledge and Research Capacity, in the ICT project BRICKS (http://www.bsik-bricks.nl), theme MSV1.

http://www.siam.org/journals/sisc/29-1/63761.html

${ }^{\dagger}$ Delft University of Technology, Faculty of Electrical Engineering, Mathematics and Computer Science, Mekelweg 4, 2628 CD Delft, The Netherlands (Ariel.Almendral.Vazquez@nr.no, c.w.oosterlee@math.tudelft.nl). 
Due to the close link between the stochastic martingale approach and the PDE approach $[24,30]$ the field of computational finance has gained a tremendous impulse from well-established numerical techniques for PDEs. For an overview we refer the reader to the introductory books [26,37]. The development of analogous reliable techniques under Lévy markets is a subject of present research $[5,6,19]$ for jumpdiffusions and for the infinite activity case $[3,4,16,27,32]$. In these papers the numerical solution of a partial integro-differential equation (PIDE) has been addressed as a tool to calculate option prices.

When valuing and risk managing exotic derivatives, practitioners demand fast and accurate prices and sensitivities. Aside from nonstandard exotic derivatives, plain vanilla options in many stock markets are actually of the American (i.e., early exercise) type, for which no analytic solutions exist. As any pricing and risk management system has to be able to calibrate to these plain vanilla options; it is of the utmost importance to be able to value these American options quickly and accurately. This paper deals with a fast, accurate evaluation of options when the underlying process is a Carr-Geman-Madan-Yor (CGMY) Lévy process of infinite activity and finite variation, considered in finance in [13]. In previous work [3, 4], we computed the integral term in the PIDE by the trapezoidal rule, a choice that produced a firstorder accurate overall convergence. Our contribution here is to show that second-order convergence may be obtained for a large range of the problem parameters $(0<\alpha<1$ in (2) below).

The approach in this paper differs from existing methods in the literature. We use an integration by parts technique to rewrite the integro-differential operator in terms of Volterra operators with a weakly singular kernel. The vast classical numerical literature on this type of operator $[10,11,31]$ proves very useful to set up a high-order discretization method. Here we focus on one particular method that has been thoroughly tested in [11], namely, the collocation method for Volterra equations. The integro-differential operator in this new setting is not in the standard form in the literature, so the techniques need to be adapted to this particular example. The computation of integrals is expensive, since the number of operations involved is in general of order $O\left(N^{2}\right)$. With the help of the fast Fourier transform (FFT) algorithm it is possible to speed up computations to gain an almost linear complexity $(O(N \log N))$, provided the grid employed is uniform. The method proposed allows the application of the FFT algorithm. In the context of weakly singular Volterra (integro-differential) equations, this has been implemented in [23].

The paper is organized as follows. Section 2 offers a brief introduction to financial derivatives under exponential Lévy models together with the integro-differential equations of interest. Section 3 contains the core of the paper: the transformation of the equation by integration by parts, the collocation method on the resulting weakly singular Volterra equation, and a time integration method. A series of numerical experiments is presented in section 4, confirming the second-order accuracy of the discretization. One of the advantages of the collocation method is that one can deal with complicated payoffs, e.g., for butterfly options, straddles, etc., without affecting the accuracy. The numerical experiments in section 4 will confirm this statement. Technical details regarding the discretization are placed in appendices. A stability analysis for a model problem is also given in an appendix.

2. The exponential Lévy model in finance. Let us briefly introduce the exponential Lévy model. By a Lévy process $\left\{L_{t}\right\}_{t \geq 0}$ we mean any process starting at the origin, with stationary, independent increments. We restrict ourselves to those 
Lévy processes that can be expressed as

$$
L_{t}=(r-q+\zeta) t+\sigma W_{t}+Z_{t},
$$

where $r \geq 0$ and $q \geq 0$ denote the risk-free interest rate and the continuous dividend paid by some asset $S_{t}$, respectively. The parameter $\sigma$ denotes the volatility. This process has a drift term controlled by $\zeta$, a Brownian component $\left\{W_{t}\right\}_{t \geq 0}$, and a purejump component $\left\{Z_{t}\right\}_{t \geq 0}$. We focus on the case where the Lévy measure associated to $Z_{t}$ (see [16]) can be written as $d \nu(x)=k(x) d x$, where the weight $k(x)$ is defined as

$$
k(x)= \begin{cases}C \frac{\exp (-G|x|)}{|x|^{1+\alpha}} & \text { if } \quad x<0, \\ C \frac{\exp (-M|x|)}{|x|^{1+\alpha}} & \text { if } \quad x>0\end{cases}
$$

for constants $C>0, G \geq 0, M \geq 0$, and $\alpha<2$. The process $\left\{Z_{t}\right\}_{t \geq 0}$ is known in the literature as the CGMY process [13]; it generalizes a jump-diffusion model by Kou [29] $(\alpha=-1)$ and the Variance Gamma (VG) process [15] $(\alpha=0)$. The CGMY process is in turn a particular case of the Kobol process studied in [9], where the constant $C$ is allowed to take on different values on the positive and negative semiaxes. For convenience of notation, we have chosen parameter $\alpha$ instead of the $Y$ in [13].

2.1. Option pricing under the CGMY process. Let a market consist of one risky asset $\left\{S_{t}\right\}_{t \geq 0}$ and a bank account $\left\{B_{t}\right\}_{t \geq 0}$. Assume that the asset process $\left\{S_{t}\right\}_{t \geq 0}$ evolves according to the geometric law

$$
S_{t}=S_{0} \exp \left(L_{t}\right)
$$

where $\left\{L_{t}\right\}_{t \geq 0}$ is the Lévy process defined in (1), and the bank account follows the law $B_{t}=\exp (r t)$.

The standard tool to assign prices to options under Lévy processes consists in changing to a convenient probability measure and taking the expectation of the discounted prices. The new probability measure $Q$ is known in the financial literature as the equivalent martingale measure (EMM). It has the same null sets as the market probability, and the discounted process $\left\{e^{-(r-q) t} S_{t}\right\}_{t \geq 0}$ becomes a martingale. The so-called EMM condition $E_{Q}\left[S_{t}\right]=S_{0} e^{t(r-q)}$ together with the formula for the characteristic exponent of $L_{t}[16]$,

$$
\left.E_{Q}\left[\exp \left(L_{t}\right)\right]=\exp \left\{t\left[(r-q+\zeta)+\sigma^{2} / 2+\int_{\mathbb{R}}\left(e^{x}-1\right) k(x) d x\right)\right]\right\},
$$

implies the following "risk-neutral" form for $\zeta$ (denoted by $\varpi)$ :

$$
\varpi+\sigma^{2} / 2+\int_{\mathbb{R}}\left(e^{x}-1\right) k(x) d x=0 .
$$

Techniques used in Appendix A produce the following expression for $\varpi$ :

$$
\varpi=-\frac{\sigma^{2}}{2}-C \Gamma(-\alpha)\left\{(M-1)^{\alpha}-M^{\alpha}+(G+1)^{\alpha}-G^{\alpha}\right\}
$$


with $\Gamma(\cdot)$ the gamma function. Note that $\zeta$ controls the drift of the market process, whereas $\varpi$ is a new parameter controlling the drift in the artificial world described by the new probability measure $Q$, under which options can be priced straightforwardly. The same notation is kept for the risk-neutral parameters $G$ and $M$. The other parameters $\sigma, C$, and $\alpha$ remain unchanged when passing to the $Q$ measure; see, e.g., [16]. $M$ must be larger than one for $\varpi$ to be well defined.

European vanilla options. Consider a European option on the asset $\left\{S_{t}\right\}_{t \geq 0}$, with maturity time $T$, and strike price $K$. Let $\hat{u}(s, t)$ denote its price, with $t$ denoting the time to expiration. It is well known that $\hat{u}$ satisfies a PIDE; see, for example, [16]. In order to write a convenient expression for the PIDE, let $s=\exp (x)$ and consider the log-prices $u(x, t)$. Then in general $u$ solves the Cauchy problem

$$
\left\{\begin{array}{l}
u_{t}-\mathcal{L} u=0, \quad t \in(0, T], \quad x \in \mathbb{R}, \\
u(x, 0)=\psi(x), \quad x \in \mathbb{R},
\end{array}\right.
$$

where $\mathcal{L}$ is an integro-differential operator of the form

$$
\begin{aligned}
\mathcal{L} \varphi:=\frac{\sigma^{2}}{2} \varphi_{x x} & +\left(r-q-\frac{\sigma^{2}}{2}\right) \varphi_{x}-r \varphi \\
& +\int_{\mathbb{R}}\left[\varphi(x+y, t)-\varphi(x, t)-\left(e^{y}-1\right) \varphi_{x}(x, t)\right] k(y) d y,
\end{aligned}
$$

and the so-called payoff function $\psi$ defines the nature of the contract. For example, for a put option $\psi(x)=\left(K-e^{x}\right)^{+}$, meaning that the holder of the option is entitled to receive the payment $K-S_{T}$ if the asset value falls below the threshold $K$; otherwise the option expires worthless.

For the particular case under consideration, namely, $\alpha<1$, the third term in the integral operator may be computed explicitly (cf. (5)), resulting in the expression

$$
\mathcal{L} \varphi:=\frac{\sigma^{2}}{2} \varphi_{x x}+(r-q+\varpi) \varphi_{x}-r \varphi+\int_{\mathbb{R}}[\varphi(x+y, t)-\varphi(x, t)] k(y) d y .
$$

This operator reduces to the Black-Scholes operator if no jumps are present, i.e., $k \equiv 0$.

American vanilla options. Consider as a matter of example an American put option written on the underlying asset $\left\{S_{t}\right\}_{t \geq 0}$. It is also well known that its price may be found by solving the free-boundary problem [16]

$$
\left\{\begin{array}{l}
u_{t}-\mathcal{L} u=0, \quad t>0, \quad x>x_{f}(t), \\
u(x, t)=K-e^{x}, \quad t>0, \quad x \leq x_{f}(t), \\
u(x, t) \geq\left(K-e^{x}\right)^{+}, \quad t>0, \quad x \in \mathbb{R}, \\
u_{t}-\mathcal{L} u \geq 0, \quad t>0, \quad x \in \mathbb{R}, \\
u(0, x)=\left(K-e^{x}\right)^{+}, \quad x \in \mathbb{R},
\end{array}\right.
$$

where the operator $\mathcal{L}$ is defined in (8) and the free-boundary is given by

$$
x_{f}(t)=\inf \left\{x \in \mathbb{R} \mid u(x, t)>\left(K-e^{x}\right)^{+}\right\}, \quad t \in(0, T] .
$$


The set $\left\{x \in \mathbb{R} \mid x \leq x_{f}(t)\right\}$ represents the exercise region for the logarithmic prices. Hence, for asset prices $s \leq s_{f}(t):=\exp \left(x_{f}(t)\right)$, the American put should be exercised.

For numerical purposes, a useful reformulation of (10) is the following linear complementarity problem (LCP):

$$
\begin{cases}u_{t}-\mathcal{L} u \geq 0 & \text { in }[0, T] \times \mathbb{R}, \\ u \geq \psi & \text { in }[0, T] \times \mathbb{R}, \\ \left(u_{t}-\mathcal{L} u\right)(u-\psi)=0 & \text { in }[0, T] \times \mathbb{R}, \\ u(x, 0)=\psi(x) . & \end{cases}
$$

This formulation does not make use of the free-boundary explicitly, and besides, it does not rely on the form of the continuation region. The free-boundary can be obtained in a postprocessing step by using the relation (11).

\section{Discretization of the integral term.}

3.1. Integral equation over the positive semiaxis. The core of the numerical method that we propose here lies on a proper discretization of integral expressions of the form

$$
f(x)=\int_{0}^{\infty}(u(x+y)-u(x)) \frac{e^{-M y}}{y^{1+\alpha}} d y
$$

that appear in the definition of $\mathcal{L}$ in (9). For ease of notation we have omitted the time variable. We suppose that $u$ is known and we would like to approximate $f(x)$. The reason for not solving with respect to $u$ in the first place is that we will use an explicit time-stepping scheme for solving (7). In the applications, the function $u(x)$ can be continuous but need not be differentiable at certain points.

Integration by parts of (13) yields

$$
f(x)=-\left.[u(x+y)-u(x)] \hat{k}(y)\right|_{0} ^{\infty}+\int_{0}^{\infty} u_{y}(x+y) \hat{k}(y) d y,
$$

where we have introduced the function

$$
\hat{k}(w):=\int_{w}^{\infty} \frac{e^{-M \zeta}}{\zeta^{1+\alpha}} d \zeta, \quad w>0 .
$$

This new kernel may be written in terms of the upper incomplete gamma function $\Gamma(\cdot, \cdot)$; see (50) in Appendix A for a definition and also [1] for some properties. The following expression holds:

$$
\hat{k}(w)=M^{\alpha} \Gamma(-\alpha, M w) .
$$

Under rather general conditions on the function $u(x)$, the first term in (14) vanishes. A possible set of sufficient conditions is

- The function $u$ satisfies the Lipschitz condition; i.e., there is a constant $C_{0}$ such that

$$
\left|u\left(z_{1}\right)-u\left(z_{2}\right)\right| \leq C_{0}\left|z_{1}-z_{2}\right| \quad \forall z_{1}, z_{2} \in \mathbb{R} .
$$


- There exist constants $C_{1} \geq 0$ and $0 \leq C_{2}<M$ such that

$$
|u(z)| \leq C_{1} e^{C_{2} z}, \quad z \rightarrow+\infty .
$$

If these two conditions hold, it is not difficult to show that

$$
\lim _{y \rightarrow 0}[u(x+y)-u(x)] \hat{k}(y)=\lim _{y \rightarrow \infty}[u(x+y)-u(x)] \hat{k}(y)=0 .
$$

For commonly used functions in option pricing like $u(x)=\left(K-e^{x}\right)^{+}$and $u(x)=$ $\left(e^{x}-K\right)^{+}$, both conditions above hold.

Changing variable $z=x+y$, integral equation (14) reduces to a Volterra equation of the first kind:

$$
f(x)=\int_{x}^{\infty} u_{z}(z) \hat{k}(z-x) d z .
$$

This kind of equation has been intensively studied in the last century, both theoretically and numerically; see $[10,11,31]$ and the references therein. In this paper we follow very closely the notation in [11].

3.1.1. Collocation method on positive semiaxis. The first observation concerns the order of the singularity of the kernel $\hat{k}$. It is not difficult to realize that $\hat{k}$ behaves like $y^{-\alpha}$, as $y \rightarrow 0$. Hence, the integral equation (20) classifies as a weakly singular Volterra equation.

Motivated by the above observations, let us introduce a notation for a new kernel $\widetilde{k}$ and the derivative $v$ of $u$, i.e.,

$$
\widetilde{k}(w):=w^{\alpha} \hat{k}(w), \quad v:=u_{z} .
$$

From now on we focus on the discretization of the integral equation

$$
f(x)=\int_{x}^{\infty}(z-x)^{-\alpha} v(z) \widetilde{k}(z-x) d z,
$$

following [11] closely. In this form it resembles the well-studied Abel equation [31]. In the examples considered in this paper, the functions $u$ have a rapidly decaying derivative at infinity. Thus, one may truncate this integral to a finite interval $x \in[a, b]$ while having control of the error. An analysis of the size of the truncation domain is given in section 3.1.3 and a numerical test is presented in section 3.1.4.

Let us consider the following (not necessarily uniform) spatial mesh

$$
x_{0}=a ; \quad x_{n+1}=x_{n}+h_{n}, \quad n=0, \ldots, N-1 ; \quad x_{N}=b .
$$

Equation (22) is discretized by the collocation method. A discretization with two collocation points for each mesh point is considered here. For a more general treatment we refer the reader to [11].

The first step is to choose two numbers $c_{1}$ and $c_{2}$ such that

$$
0 \leq c_{1}<c_{2}<1
$$

With these constants the collocation mesh is defined as follows (see Figure 1):

$$
x_{n, j}=x_{n}+c_{j} h_{n}, \quad n=0, \ldots, N-1 ; \quad j=1,2 .
$$




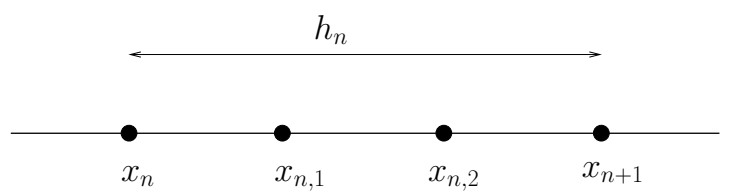

FIG. 1. Collocation mesh and spatial mesh.

The polynomial spline collocation method now consists, roughly speaking, in finding a polynomial spline $V(z)$, replacing the function $v(z)$ such that (22) holds exactly at each of the collocation points $x_{n, j}$. The technical details of the treatment of the Volterra equation when the variable of integration runs through the positive semiaxis are presented in Appendix B. For the negative semiaxis the discretization is very similar and is explained in Appendix C. Considering the collocation parameters $0<$ $c_{1}<c_{2} \leq 1$, the collocation mesh for the negative semiaxis is defined as in (23); however, these two meshes are typically different. This time $c_{1} \neq 0$. In section 3.2 we couple both integrals with the use of a common spatial mesh.

REMARK 3.1. The convergence of polynomial spline collocation methods for firstkind Volterra integral equations with integrable algebraic kernel singularities is still an open problem; see, e.g., [22] and [10, Chapter 6]. The convergence properties of these collocation methods, like for the nonstandard weakly singular Volterra equation (22) (integration from $x$ to $\infty$ ) have not yet been studied at all. We will present numerical experiments showing that two-point collocation based on the collocation parameters $c_{1}$ and $c_{2}$ yield convergent schemes. The theoretical foundation for this is, however, still lacking.

3.1.2. Smoothing the kernel. A simple numerical experiment with (22) reveals that the order of the discretization is only $O(h)$ (cf. Table 1). At first glance it seems that the problem is caused by the nonsmooth payoff. However, the cause of this loss of accuracy is the lack of smoothness of the kernel $\widetilde{k}$. This can be easily remedied by a convenient rewriting of the integral equation. One possible remedy is based on an expansion of the kernel such that the nonsmooth component can be identified. We will add a subscript "+" to the kernel to indicate that we work with the positive of the Lévy measure. A first integration by parts yields

$$
\hat{k}(x)=\int_{x}^{\infty} \frac{e^{-M y}}{y^{1+\alpha}} d y=\frac{e^{-M x} x^{-\alpha}}{\alpha}-\frac{M}{\alpha} \int_{x}^{\infty} e^{-M y} y^{-\alpha} d y .
$$

A second integration by parts for the integral on the right-hand side gives rise to the identity

$$
\int_{x}^{\infty} \frac{e^{-M y}}{y^{1+\alpha}} d y=\frac{e^{-M x} x^{-\alpha}}{\alpha}+\frac{M e^{-M x} x^{1-\alpha}}{\alpha(1-\alpha)}-\frac{M^{2}}{\alpha(1-\alpha)} \int_{x}^{\infty} e^{-M y} y^{1-\alpha} d y .
$$

So, if we define the constant

$$
\theta_{+}:=\frac{M^{2}}{\alpha(1-\alpha)} \int_{0}^{\infty} e^{-M y} y^{1-\alpha} d y
$$

the new kernel

$$
k_{+}(x):=\widetilde{k}(x)+\theta_{+} x^{\alpha}=\frac{e^{-M x}}{\alpha}+\frac{M e^{-M x} x}{\alpha(1-\alpha)}-\frac{M^{2} x^{\alpha}}{\alpha(1-\alpha)} \int_{0}^{x} e^{-M y} y^{1-\alpha} d y
$$


becomes a differentiable function, in contrast to the original kernel $\widetilde{k}(x)$ in $(21)$ which is only continuous. Moreover, due to the second integration by parts, $k_{+}$is even twice continuously differentiable. Indeed, differentiating twice the relevant term on the right-hand side and neglecting the smooth terms give

$$
x^{\alpha-2} \int_{0}^{x} e^{-M y} y^{1-\alpha} d y
$$

which is continuous, but not differentiable. A final observation is that $\theta_{+}$may be written in terms of the gamma function $\Gamma(\cdot)$; i.e.,

$$
\theta_{+}=\frac{M^{\alpha} \Gamma(2-\alpha)}{\alpha(1-\alpha)}
$$

The observations lead to the integral equation

$$
f(x)=\int_{x}^{\infty}(z-x)^{-\alpha} v(z) k_{+}(z-x) d z-\theta_{+} \int_{x}^{\infty} v(z) d z .
$$

Notice that (27) is merely a rewriting of (22), but the kernels present in (27) are smoother. One could continue integrating by parts and extract the smooth components of $\widetilde{k}$. For numerical efficiency this is however not advisable as each additional term means a new integral to be computed numerically, increasing therefore the complexity. In order to avoid truncation errors we treat both terms on the right-hand side in (27) numerically.

3.1.3. Analysis of the truncation error. We sketch an argument that provides an estimate for the truncation error. The details have been omitted for clarity.

It is known that the solution $u$ of (7) admits the stochastic interpretation

$$
u(x, t)=e^{-r t} E_{Q}\left[\psi\left(x+L_{t}\right)\right] .
$$

Consider the case of a put option, i.e., $\psi(x)=\left(K-e^{x}\right)^{+}$. Denoting by $\hat{q}(y)$ the probability density function of the variable $L_{t}$, we may write

$$
u(x, t)=e^{-r t} \int_{-\infty}^{\log K-x}\left(K-e^{x+y}\right) \hat{q}(y) d y .
$$

Differentiating this expression with respect to $x$ yields

$$
u_{x}(x, t)=-e^{-r t+x} \int_{-\infty}^{\log K-x} e^{y} \hat{q}(y) d y .
$$

Since $e^{y} \leq K e^{-x}$ for $y \leq \log (K)-x$, the following estimate holds:

$$
\left|u_{x}(x, t)\right| \leq K e^{-r t} .
$$

This is a global estimate. A more detailed analysis shows that the derivative of a put decays exponentially, giving a sharper estimate. For our purposes this bound is sufficient. An estimate for the truncation error may be obtained as follows:

$$
\begin{aligned}
\mid \int_{-\infty}^{a} u_{z}(z, t) \hat{k}_{-}(x-z) d z & +\int_{b}^{+\infty} u_{z}(z, t) \hat{k}_{+}(z-x) d z \mid \\
& \leq K e^{-r t}\left[\int_{-\infty}^{a} \hat{k}_{-}(x-z) d z+\int_{b}^{+\infty} \hat{k}_{+}(z-x) d z\right] .
\end{aligned}
$$


Both integrals on the right-hand side have exponential decay as $a \rightarrow-\infty$ and $b \rightarrow$ $+\infty$. Namely, from (15), a rough estimate is the following:

$$
\hat{k}_{+}(w) \leq C e^{-M w} .
$$

Therefore, the second integral on the right-hand side may be bounded by

$$
\frac{C^{+}}{M} e^{-M(b-x)} .
$$

An analogous analysis shows that the first integral is controlled by

$$
\frac{C^{-}}{G} e^{-G(x-a)}
$$

These estimates show that, away from the end points $a$ and $b$, the error decreases exponentially. Close to the end points the exponential decay of the derivative $u_{x}$ (for a put option) will play a role in reducing the error by truncation.

3.1.4. First numerical experiments. In this section we first show that the collocation method applied to (27) gives at least a quadratic discretization error. We choose the payoff function for a put option $u(x)=\left(1-e^{x}\right)^{+}$and compare it with the analytic formula for $f(x)$ derived in Appendix A (52). Moreover, we check that indeed the same method applied to (22) gives only a linear order, so that the smoothing of the integrand as explained in section 3.1.2 is necessary for second-order accuracy. The results are displayed in Table 1 . The parameters used are $\alpha=0.8$ and $M=5$ and the mesh is uniform. The truncation of the domain is in accordance with the analysis in section 3.1.3. In particular, the computational domain in this example is the interval $[-5,5]$. The truncation does not influence the accuracy negatively.

The same convergence results hold on a nonuniform grid, but we are then not able to apply the FFT algorithm (discussed in section 3.4 and in detail in [3]) to make computations fast. On the other hand, the discretization order depends on the choice of the collocation parameters $c_{1}$ and $c_{2}$, as Table 1 shows. We actually observe superconvergence at the collocation points with the Radau parameters $c_{1}=0$ and $c_{2}=2 / 3$. This is in accordance with the results from [11].

TABLE 1

Discretization errors with the collocation method.

\begin{tabular}{|c|c|c||c|c||c|c|}
\hline & \multicolumn{3}{|c||}{ Eq. $(27)$} & \multicolumn{2}{c|}{ Eq. $(22)$} \\
\hline & \multicolumn{2}{|c||}{$c_{1}=0, c_{2}=0.5$} & \multicolumn{2}{|c|}{$c_{1}=0, c_{2}=2 / 3$} & \multicolumn{2}{c|}{$c_{1}=0, c_{2}=0.5$} \\
\hline$N$ & $\ell_{\infty}$-error & rate & $\ell_{\infty}$-error & rate & $\ell_{\infty}$-error & rate \\
\hline 20 & 0.0839 & & 0.02314 & & 0.248 & \\
\hline 40 & 0.0240 & 3.4 & 0.00366 & 6.3 & 0.148 & 1.6 \\
\hline 80 & 0.0065 & 3.6 & 0.00057 & 6.4 & 0.079 & 1.8 \\
\hline 160 & 0.0017 & 3.8 & 0.00009 & 6.3 & 0.041 & 1.9 \\
\hline
\end{tabular}

In order to obtain superconvergence on the negative semiaxis, the Radau points must be chosen as $c_{1}=1 / 3$ and $c_{2}=1$.

3.2. Assembling the PIDE. Recall that our main goal is to solve a PIDE of the form (7) with the operator $\mathcal{L}$ given by (9). The interesting case is when $\sigma=0$, i.e., no smoothing takes place caused by a diffusion component. In case we deal with $\sigma>0$, the ideas discussed in this paper still apply, but then special care of the diffusion needs 
to be taken by means of an implicit-explicit scheme as in $[3,4,16]$, where the diffusion part is taken implicitly and early exercise is dealt with by projection methods.

The considerations from previous sections lead us to consider the following rewriting of (7):

$$
u_{t}+r u=C \mathcal{I}_{+}\left(u_{z}\right)+C \mathcal{I}_{-}\left(u_{z}\right)+\mu u_{x}
$$

with the constant (cf. (6))

$$
\mu:=r-q+\varpi
$$

and the operators

$$
\begin{aligned}
& \mathcal{I}_{+}\left(u_{z}\right):=\int_{x}^{+\infty}(x-z)^{-\alpha} u_{z}(z) k_{+}(z-x) d z-\theta_{+} \int_{x}^{+\infty} u_{z}(z) d z, \\
& \mathcal{I}_{-}\left(u_{z}\right):=-\int_{-\infty}^{x}(x-z)^{-\alpha} u_{z}(z) k_{-}(z-x) d z+\theta_{-} \int_{-\infty}^{x} u_{z}(z) d z .
\end{aligned}
$$

Note that these operators involve both the function $u$ and its derivative $v=u_{z}$. To our knowledge, PIDEs like (32)-(35) have not been considered in the existing literature on Volterra equations.

If one applies a time integration procedure, one faces the problem of finding the numerical derivative of the unknown for a next time step, discussed in section 3.2.1. This is related to the treatment of the term $\mu u_{x}$ in a consistent way.

In (32) we make a "superposition" of two operators. The positive operator behaves essentially as a "convection term" in that the solution is "transported" in the negative direction. The negative operator behaves exactly opposite, "transporting" the solution in the positive direction.

REMARK 3.2. We observed that optimal convergence properties are attained when considering Radau points, as in Table 1. For the positive part this amounts to taking the collocation mesh $X^{+}$as $x_{n, 0}=x_{n}$ and $x_{n, 1}=x_{n}+\frac{2}{3} h_{n}$; whereas for the negative operator the Radau collocation mesh $X^{-}$is $x_{n, 0}=x_{n}+\frac{1}{3} h_{n}$ and $x_{n, 1}=x_{n+1}$.

To couple the partial results over these two different meshes we propose here a straightforward interpolation: the evaluation of the integral on the $X^{+}$and $X^{-}$ meshes is interpolated to the "base mesh" $X^{+} \cup X^{-}$. We use a simple linear interpolation, though a higher-order interpolation is in principle also applicable.

3.2.1. Local differentiation formulas due to the collocation method. There are several choices to discretize the numerical derivative in (34)-(35). The stable one is to apply once more the collocation method to the equation

$$
u(x)=-\int_{x}^{b} v(s) d s+u(b)
$$

for the positive integral. For the negative integral one should instead use the formula

$$
u(x)=\int_{a}^{x} v(s) d s-u(a) .
$$

The formulas for the negative operator were derived in [11]. We have derived them for the positive operator. The formulas are summarized below for the sake of completeness. The formulas associated to (36) are called "forward formulas," and the ones 
associated to (37) "backward formulas." As remarked in [11], to be able to obtain stable local differentiation formulas, one of the collocation points must coincide with one extreme of the interval, namely, $0=c_{1}<c_{2}<1$. For the backward formulas one must have $0<c_{1}<c_{2}=1$. One picks two arbitrary numbers $\nu_{1}, \nu_{2} \in[0,1]$, that need not be the same for the forward and backward formulas.

Forward formulas. On collocation point $x_{n, 1}$ :

$$
\begin{aligned}
V_{n, 1}=\frac{1}{h_{n} c_{2}\left(1-c_{2}\right)}\left[c_{2}\left(2 \nu_{1}-c_{2}\right) u\left(x_{n}+h_{n}\right)\right. & \\
+ & \left.\left(1-2 \nu_{1}\right) u\left(x_{n}+c_{2} h_{n}\right)+\left(\left(c_{2}\right)^{2}+2\left(1-c_{2}\right) \nu_{1}-1\right) u\left(x_{n}\right)\right]
\end{aligned}
$$

On collocation point $x_{n, 2}$.

$$
\begin{aligned}
V_{n, 2}=\frac{1}{h_{n} c_{2}\left(1-c_{2}\right)}\left[c_{2}\left(2 \nu_{2}-c_{2}\right) u\left(x_{n}+h_{n}\right)\right. & \\
+ & \left.\left(1-2 \nu_{2}\right) u\left(x_{n}+c_{2} h_{n}\right)+\left(\left(c_{2}\right)^{2}+2\left(1-c_{2}\right) \nu_{2}-1\right) u\left(x_{n}\right)\right]
\end{aligned}
$$

Backward formulas [11].

$$
\begin{aligned}
& V_{n, 1}=\frac{1}{h_{n} c_{1}\left(1-c_{1}\right)}\left[c_{1}\left(2 \nu_{1}-c_{1}\right) u\left(x_{n}+h_{n}\right)\right.\left.+\left(1-2 \nu_{1}\right) u\left(x_{n}+c_{1} h_{n}\right)+\left(\left(c_{1}\right)^{2}+2\left(1-c_{1}\right) \nu_{1}-1\right) u\left(x_{n}\right)\right] \\
& V_{n, 2}=\frac{1}{h_{n} c_{1}\left(1-c_{1}\right)}\left[c_{1}\left(2 \nu_{2}-c_{1}\right) u\left(x_{n}+h_{n}\right)\right. \\
&\left.\quad+\left(1-2 \nu_{2}\right) u\left(x_{n}+c_{1} h_{n}\right)+\left(\left(c_{1}\right)^{2}+2\left(1-c_{1}\right) \nu_{2}-1\right) u\left(x_{n}\right)\right] .
\end{aligned}
$$

We now explain the treatment of the term $\mu u_{x}$. The consistent way of discretizing this term is by taking into account the transport of information produced by each of the integral operators. We may summarize the criterion as follows.

REMARK 3.3. The term $\mu u_{x}$ is discretized according to the sign of $\mu$ : by a forward collocation derivative (formulas (38)-(39)) if $\mu>0$, or by a backward collocation derivative (formulas (40)-(41)) if $\mu<0$.

3.3. Time integration. Let $k$ be the time step size, $t_{m}=m k$, for $m=$ $0, \ldots, N_{t}$. For a European option, the explicit backward differentiation formula (BDF) method reads

$$
\frac{3}{2} u^{m}-2 u^{m-1}+\frac{1}{2} u^{m-2}+k r u^{m}=k\left[I^{+}\left(\bar{v}^{m}\right)+I^{-}\left(\bar{v}^{m}\right)+\mu \bar{v}^{m}\right],
$$

where $\bar{v}^{m}=2 v^{m-1}-v^{m-2}$, and $I^{+}, I^{-}$denote the discretizations by collocation of the operators $I_{+}$and $I_{-}$, respectively. The addition on the right-hand side needs to be properly defined, as we add quantities that are not defined over the same mesh. To give a meaning to this sum, consider the base mesh $X^{+} \cup X^{-}$, i.e., the mesh where these additions make sense. Let us consider the case of Radau points. Let $P_{+}$denote the prolongation operator from $X^{+}$to $X^{+} \cup X^{-}$. An example of such a prolongation is the following:

$$
\begin{aligned}
P_{+}(w)_{3 k} & =w_{k, 1}, \\
P_{+}(w)_{3 k+1} & =\left(w_{k, 1}+w_{k, 2}\right) / 2, \\
P_{+}(w)_{3 k+2} & =w_{k, 2}
\end{aligned}
$$


for $k=0, \ldots, N-1$. A negative prolongation $P_{-}$may be defined likewise. Then, the sum of integrals in (42) is in the sense $P_{+}\left(I^{+}\left(\bar{v}^{m}\right)\right)+P_{-}\left(I^{-}\left(\bar{v}^{m}\right)\right)$. If the collocation parameters are chosen to be $c_{1}=0, c_{2}=0.5$ for the positive situation and $c_{1}=$ $0.5, c_{2}=1$ for the negative situation, one does not need to interpolate as the meshes coincide, except for boundary points. However, this choice is less accurate as pointed out in section 3.1.4.

Each derivative $v^{m}$ present in the integrands is evaluated by the local differentiation formulas of section 3.2.1. An iteration of the form

$$
u^{m}=J\left(v^{m-1}, v^{m-2}\right)
$$

is then found after collecting the prolongation terms. The first input to this iteration is the collocation derivative of the payoff $v^{0}$, and the second, $v^{1}$, is the result of differentiating one explicit Euler iteration.

For American options the iteration is similar:

$$
\left\{\begin{array}{l}
u^{m} \geq J\left(v^{m-1}, v^{m-2}\right) \\
u^{m} \geq \psi \\
\left(u^{m}-J\left(v^{m-1}, v^{m-2}\right), u^{m}-\psi\right)=0 \\
u^{0}=\psi
\end{array}\right.
$$

Here $(\cdot, \cdot)$ is the standard inner product in $\mathbb{R}^{3 N}$, because these iterations are carried out in a mesh of size $3 N$ due to the collocation points and the size of the base mesh. The solution of this discrete LCP is simply $u^{m}=\max \left(J\left(v^{m-1}, v^{m-2}\right), \psi\right)$.

3.4. The algorithm. We summarize the techniques discussed in an algorithm. The notations $v_{f}$ and $v_{b}$ stand for the forward and backward collocation derivative, respectively, as in section 3.2.1. The operator $P_{+}(u)$ means the prolongation operator by interpolation from collocation mesh $X^{+}$to the base mesh $X^{+} \cup X^{-}$. The operator $P_{-}(u)$ defines likewise the prolongation from $X^{-}$. Finally, the term $\mu u_{x}$ is prolongated similarly and added to one of the integrals according to Remark 3.3.

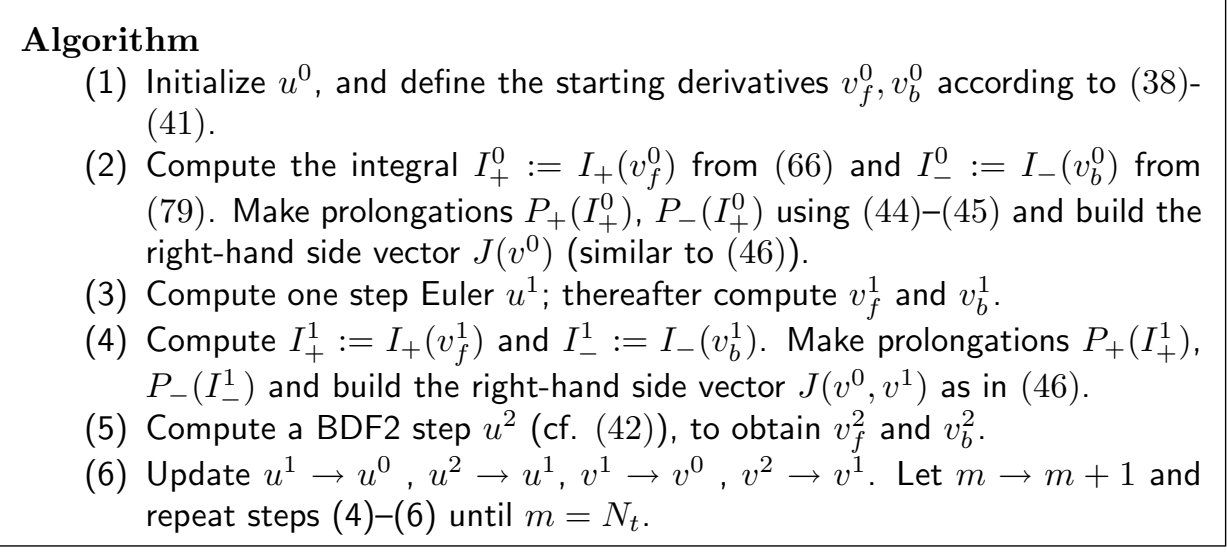

Whenever we compute the integrals $I_{+}$and $I_{-}$we apply the FFT algorithm to accelerate the computation of the convolution. This is standard practice so the details are left out. In previous work $[3,4]$ we made use of this technique to reduce computational time. We only point out that formulas (64) and (82) take on a special form 
when the mesh is uniform, representing the multiplication of a Toeplitz matrix by a vector. These matrix-vector multiplications can be efficiently implemented following the explanation in [36].

4. Numerical experiments. The experiments in this section are performed on an AMD Athlon(TM) XP 2700. The first experiment consists in computing European put options with different values of $\alpha$ in order to verify that the order of convergence is quadratic and independent of $\alpha$; see Table 2. Here, "rate" is defined as the ratio of errors. So, a factor 4 corresponds to second-order accuracy. The numerical values are compared with the Carr-Madan formula [14]. We note that with increasing $\alpha$, the number of time steps should be increased to obtain a stable solution. The numerical tests for the option pricing problems confirm that in order for the solution to exhibit a stable behavior, a stability condition $k \leq C(\alpha) h^{\alpha}$ should be fulfilled. The experiments show also that the stability constant $C(\alpha)$ decreases when $\alpha$ approaches 1 , which is in accordance with the stability analysis in Appendix D.

Since the grid convergence rate is also deteriorating with increasing $\alpha$ (Table 2), one does not expect the superconvergence for $\alpha$ close to one. However, $O\left(h^{2}\right)$ accuracy is clearly observed.

A second experiment consists in computing an American put option (Table 3). We again observe at least second-order accuracy of the scheme. An accuracy higher than two seems awkward at first sight with a second-order time integration scheme. Starting with $N=N_{t}=20$, however, and doubling both $N$ and $N_{t}$, one observes (not shown here) in fact that the explicit BDF2 collocation method is second-order in time. The higher rate observed in Table 3 is basically the effect of having a higher order in space than in time, combined with a special choice of $h$ and $k$.

The CPU times behave as predicted by the application of the FFT for each time step, with $O\left(N_{t} N \log N\right)$ complexity. Notice that the computations will be significantly more rapid if we need engineering accuracy, i.e., 3 digits accurate. Pictures of the American put option values at $t=0$ and the time-dependent early exercise boundaries for different values of parameter $\alpha$ are shown in Figure 2. Increasing $\alpha$ means an increase of the intensity of small jumps. Loosely speaking, the prices behave similarly to a volatility increase in the classical Black-Scholes framework.

In a third experiment, European and American butterfly options are considered (Table 4 and Figure 3). A butterfly spread is the result of buying call options with strikes $K_{1}$ and $K_{2}$, respectively, and selling two call options with strike price $K_{3}=$ $\left(K_{1}+K_{2}\right) / 2$. That is, the payoff is of the form

$$
\psi(x)=\left(e^{x}-K_{1}\right)^{+}-2\left(e^{x}-\frac{K_{1}+K_{2}}{2}\right)^{+}+\left(e^{x}-K_{2}\right)^{+} .
$$

A European style butterfly is appropriate when the investor thinks large moves in the price of the underlying asset are unlikely. Usually the middle strike $K_{3}$ is close to today's spot price $S_{0}$, so that it pays off when the underlying asset price stays close to $S_{0}$. In Figure 3 we show American style butterflies (in the $S$ coordinate) with different values of $\alpha$ and in Table 4 the second-order convergence of a European style butterfly is shown experimentally. The reason for including a butterfly option example is to show that the method discussed in this paper is rather general and provides accurate numerical results for other kinds of payoffs (nonconvex payoffs, for example), without any further complications.

A final remark is concerning the so-called smooth-fit or smooth-paste principle. This principle essentially states that the derivative of the Black-Scholes American 
TABLE 2

Computation of European options for different values of $\alpha$. Other parameters: $r=0.1, q=$ $0, K=1, C=1, M=G=5, T=1$.

\begin{tabular}{c|c|c|c|c|c}
\hline$\alpha$ & $N$ & $N_{t}$ & Error at $x=0$ & Rate & Ref. value \\
\hline \multirow{4}{*}{0.1} & 100 & 20 & $9.73 \mathrm{E}-4$ & & \\
& 200 & 40 & $1.82 \mathrm{E}-4$ & 5.3 & \multirow{2}{*}{0.06353404} \\
& 400 & 80 & $3.18 \mathrm{E}-5$ & 5.7 & \\
\hline \hline \multirow{4}{*}{$\alpha=0.5$} & 800 & 160 & $5.70 \mathrm{E}-6$ & 5.5 & \\
& 100 & 40 & $1.58 \mathrm{E}-3$ & & \\
& 200 & 80 & $3.26 \mathrm{E}-4$ & 4.8 & \multirow{2}{*}{0.10296690} \\
& 400 & 160 & $6.38 \mathrm{E}-5$ & 5.1 & \\
\hline \hline \multirow{3}{*}{$\alpha=0.8$} & 800 & 320 & $1.21 \mathrm{E}-5$ & 5.2 & \\
& 100 & 200 & $8.19 \mathrm{E}-3$ & & \\
& 200 & 400 & $1.96 \mathrm{E}-3$ & 4.1 & \multirow{2}{*}{0.14789424} \\
& 400 & 800 & $4.47 \mathrm{E}-4$ & 4.3 & \\
\hline
\end{tabular}

TABLE 3

Grid convergence for an American put. Parameters: $r=0.1, q=0, K=1, C=1, M=G=$ $5, \alpha=0.5$.

\begin{tabular}{c|c|c|c|c}
\hline$N$ & $N_{t}$ & error at $x=0$ & rate & CPU-time $(\mathrm{s})$ \\
\hline 100 & 40 & $1.73 \mathrm{E}-3$ & & 0.76 \\
\hline 200 & 80 & $3.36 \mathrm{E}-4$ & 5.1 & 1.3 \\
\hline 400 & 160 & $4.80 \mathrm{E}-5$ & 7 & 5.28 \\
\hline 800 & 320 & $6.80 \mathrm{E}-6$ & 7 & 21.10 \\
\hline ref. value & 0.112171 & \multicolumn{3}{|c}{} \\
\hline
\end{tabular}

TABLE 4

Computation of a European butterfly with parameters: $r=0.1, q=0, K=1, T=1, C=1$, $G=3, M=5, \alpha=0.5$.

\begin{tabular}{c|c|c|c}
\hline$N$ & $N_{t}$ & $\ell_{\infty}$-errors & rate \\
\hline 50 & 30 & $1.22 \mathrm{E}-2$ & \\
\hline 100 & 60 & $3.22 \mathrm{E}-3$ & 3.8 \\
\hline 200 & 120 & $5.94 \mathrm{E}-4$ & 5.4 \\
\hline 400 & 240 & $6.28 \mathrm{E}-5$ & 9.4 \\
\hline
\end{tabular}

option price is a continuous function, also at the exercise boundary. It is known that this property does not generally hold true when the underlying follows a Lévy process (for a partial proof see [4] and for a complete characterization for perpetual options see [2]). The butterfly payoff has the feature of combining both the put and the call properties. We observe on the left-hand side of the "hat payoff" in Figure 3 (the call part) that the solution enters the payoff smoothly, whereas, for the right-hand side the smooth-fit is not observable. In this example two free-boundaries appear and despite the continuous dividend yield $q=0$. This is in contrast to the well-known BlackScholes situation regarding the call option for which early exercise is not favorable with $q=0$.

5. Conclusions. In this paper we have proposed a numerical procedure to solve integro-differential scalar equations that arise in finance, when pricing options for which the underlying asset follows a Lévy process. The main idea is to integrate by parts in order to transform the integral operator into a sum of Volterra operators with weakly singular kernels. Known numerical techniques like the collocation method for Volterra equations with weakly singular kernels prove useful to set up a high-order 

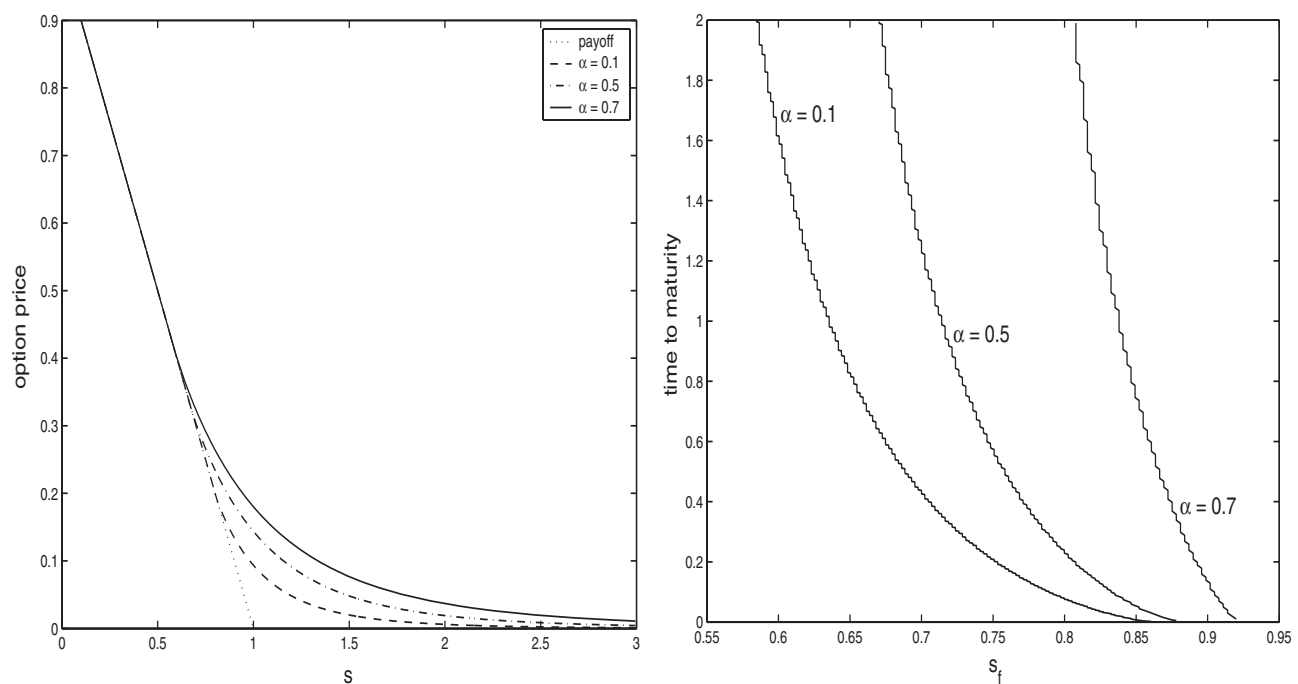

FIG. 2. American put option prices at $t=0$ (left) and early exercise boundaries (right) for different values of $\alpha$. Other parameters: $r=0.1, q=0, K=1, T=2, C=1, G=5, M=5$.
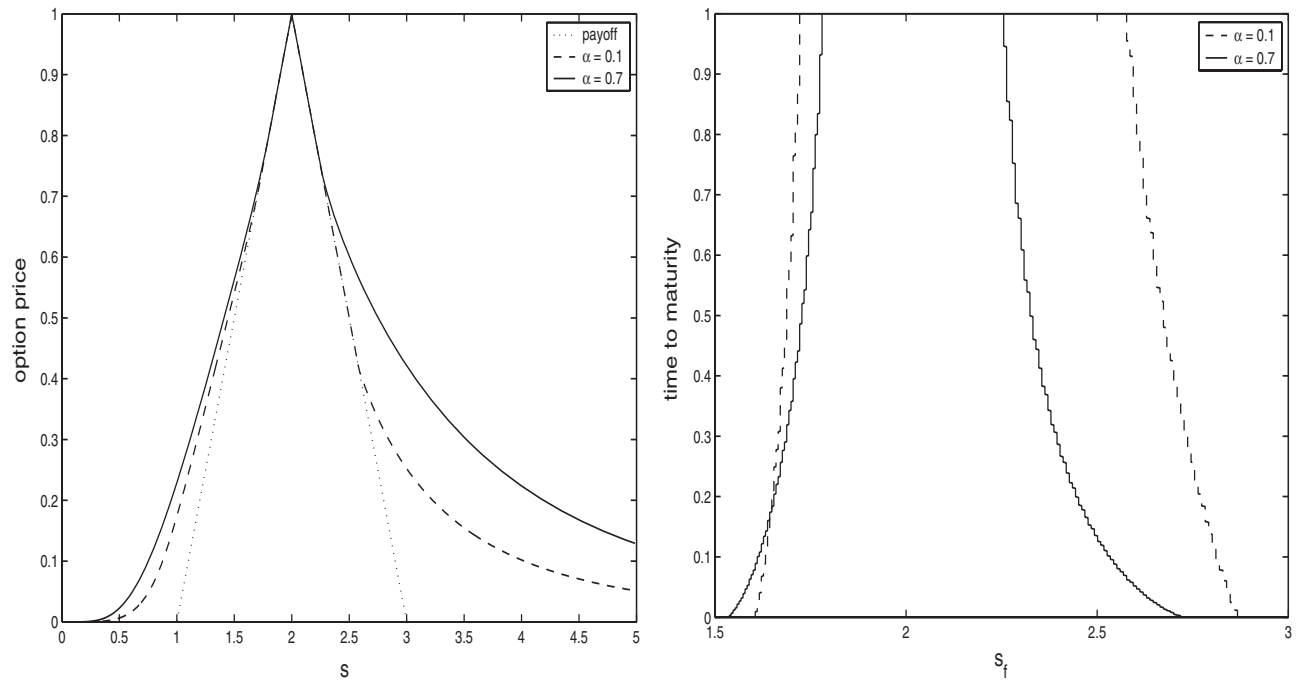

FIG. 3. American butterfly prices at $t=0$ (left) and early exercise boundaries for $\alpha=0.1$ and $\alpha=0.7$. Other parameters: $r=0.1, q=0, K_{1}=1, K_{2}=3, T=1, C=1, G=3, M=5$.

discretization. Throughout a series of experiments it has been shown that an explicit method is a natural choice for these kinds of problems and for parameter $\alpha \leq 0.7$, as they essentially behave like transport equations. The method proposed can deal with complicated payoffs in a natural way. Due to the FFT algorithm, the complexity of the method is close to linear. With a fast, second-order method at hand, the calibration problem for American options under Lévy processes is now within reach. 
Appendix A. Analytic evaluation of integral equation (13). In the numerical experiments in section 3.1.4 we make use of the analytic expression for

$$
f(x)=\int_{0}^{\infty}(u(x+y)-u(x)) \frac{e^{-M y}}{y^{1+\alpha}} d y,
$$

where $u(x)=\left(1-e^{x}\right)^{+}$. In this paragraph we derive an expression for $f(x)$.

Note that $f(x)=0$ for $x>0$. For $x<0$ we have

$$
\begin{aligned}
& \int_{0}^{+\infty}\left[\left(1-e^{x+y}\right)^{+}-\left(1-e^{x}\right)^{+}\right] \frac{e^{-M y}}{y^{1+\alpha}} d y \\
& =e^{x} \int_{0}^{-x}\left(1-e^{y}\right) \frac{e^{-M y}}{y^{1+\alpha}} d y+\left(e^{x}-1\right) \int_{-x}^{+\infty} \frac{e^{-M y}}{y^{1+\alpha}} d y \\
& =e^{x} \int_{0}^{+\infty}\left(1-e^{y}\right) \frac{e^{-M y}}{y^{1+\alpha}} d y+e^{x} \int_{-x}^{+\infty} \frac{e^{-(M-1) y}}{y^{1+\alpha}} d y-\int_{-x}^{+\infty} \frac{e^{-M y}}{y^{1+\alpha}} d y .
\end{aligned}
$$

To compute the first term, observe that for $\alpha<0$ the formula

$$
\int_{0}^{\infty} \frac{e^{-M y}}{y^{1+\alpha}} d y=M^{\alpha} \Gamma(-\alpha)
$$

holds. Therefore,

$$
\int_{0}^{+\infty}\left(1-e^{y}\right) \frac{e^{-M y}}{y^{1+\alpha}} d y=\Gamma(-\alpha)\left[M^{\alpha}-(M-1)^{\alpha}\right] .
$$

However, this formula also admits an extension to the complex plane excluding $\alpha \neq$ $1,2, \ldots ;$ see $[1]$. The second and the third term in (48) can be computed with some quadrature rule, but here we opt for the following approach. These integrals can be written in terms of the so-called upper incomplete gamma function:

$$
\Gamma(\beta, y)=\int_{y}^{+\infty} t^{\beta-1} e^{-t} d t
$$

That is, for instance,

$$
\int_{-x}^{+\infty} \frac{e^{-M y}}{y^{1+\alpha}} d y=M^{\alpha} \Gamma(-\alpha,-M x)
$$

An efficient algorithm for computing the incomplete gamma function is discussed in [38].

To summarize, we found that $f(x)=0$ for $x>0$ and

$$
\begin{aligned}
f(x)=e^{x} \Gamma(-\alpha)\left[M^{\alpha}-(M-1)^{\alpha}\right] & \\
+ & e^{x}(M-1)^{\alpha} \Gamma(-\alpha,-(M-1) x)-M^{\alpha} \Gamma(-\alpha,-M x), \quad x<0 .
\end{aligned}
$$

Appendix B. Collocation method on positive semiaxis. Here, we discretize (22) in the interval $x \in[a, b]$. One looks for a piecewise linear function, defined as $V_{l}$ on each interval $\left[x_{l}, x_{l+1}\right]$, such that

$$
f_{n, j}=\int_{x_{n, j}}^{b}\left(z-x_{n, j}\right)^{-\alpha} V(z) \widetilde{k}\left(z-x_{n, j}\right) d z .
$$




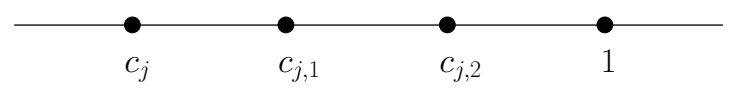

FIG. 4. Interpolation points.

The notation $f_{n, j}$ means the values $f\left(x_{n, j}\right)$.

Splitting the integral term gives the sum

$$
f_{n, j}=A_{n, j}+P_{n, j}, \quad n=0, \ldots, N-1, \quad j=1,2,
$$

where the first term is the integral from collocation point $x_{n, j}$ until the next mesh point $x_{n+1}$ :

$$
A_{n, j}:=\int_{x_{n, j}}^{x_{n+1}}\left(z-x_{n, j}\right)^{-\alpha} V_{n}(z) \widetilde{k}\left(z-x_{n, j}\right) d z ; \quad j=1,2,
$$

and the "lag term" $P_{n, j}$ takes into account the intervals $\left[x_{l}, x_{l+1}\right]$ :

$$
P_{n, j}:=\sum_{l=n+1}^{N-1} \int_{x_{l}}^{x_{l+1}}\left(z-x_{n, j}\right)^{-\alpha} V_{l}(z) \widetilde{k}\left(z-x_{n, j}\right) d z ; \quad j=1,2 .
$$

With the change of variables $z=x_{l}+\nu h_{l}$, both $A_{n, j}$ and $P_{n, j}$ transform, respectively, into

$$
A_{n, j}=h_{n}^{1-\alpha} \int_{c_{j}}^{1}\left(\nu-c_{j}\right)^{-\alpha} V_{n}\left(x_{n}+\nu h_{n}\right) \widetilde{k}\left(h_{n}\left(\nu-c_{j}\right)\right) d \nu
$$

and

$$
P_{n, j}=\sum_{l=n+1}^{N-1} h_{l}^{1-\alpha} \int_{0}^{1}\left[\frac{x_{l}-x_{n, j}}{h_{l}}+\nu\right]^{-\alpha} V_{l}\left(x_{l}+\nu h_{l}\right) \widetilde{k}\left(x_{l}-x_{n, j}+\nu h_{l}\right) d \nu .
$$

B.1. Product integration formulas on positive semiaxis. Formulas (57) and (58) are not suitable for straightforward evaluation. One would rather prefer some numerical approximation that keeps the order of accuracy. One possibility is to use an interpolatory quadrature rule.

Let us start by fixing two interpolation points belonging to the interval $\left[c_{j}, 1\right)$ :

$$
c_{j, p}:=c_{j}+\left(1-c_{j}\right) c_{p}, \quad j, p=1,2 .
$$

In other words, the same collocation points $c_{p}$ are used through a linear mapping of the interval $[0,1]$ (where they are located) into the interval $\left[c_{j}, 1\right.$ ); see [11]. Figure 4 illustrates the relative positions of these points. Let $L_{j, p}$ represent the Lagrange interpolation polynomials associated to the points $c_{j, p}$. The product integration rule consists in approximating the integrand (except for the singular part) by a piecewise linear polynomial, i.e., one employs in (57) the following approximation:

$$
V_{n}\left(x_{n}+\nu h_{n}\right) \widetilde{k}\left(h_{n}\left(\nu-c_{j}\right)\right) \approx \sum_{p=1}^{2} L_{j, p}(\nu) V_{n}\left(x_{n}+c_{j, p} h_{n}\right) \widetilde{k}\left(h_{n}\left(c_{j, p}-c_{j}\right)\right)
$$


for $\nu \in\left[c_{j}, 1\right]$. This formula requires the values $V_{n}\left(x_{n}+c_{j, p} h_{n}\right)$. Let $L_{q}$ and $V_{n, q}$ denote the Lagrange interpolation polynomials at $x_{n, q}$ for $q=1,2$ and the nodal values of $V_{n}$ at the same collocation points, respectively. It follows that

$$
V_{n}\left(x_{n}+c_{j, p} h_{n}\right)=\sum_{q=1}^{2} L_{q}\left(c_{j, p}\right) V_{n, q}, \quad j=1,2 .
$$

The function $V(z)$ need not be continuous. Upon substitution of (60) into (59) and thereafter into (57), one arrives at the formulas suitable for numerical evaluation,

$$
\begin{aligned}
& \hat{A}_{n, j}=h_{n}^{1-\alpha} \sum_{p=1}^{2} \sum_{q=1}^{2} \lambda_{j, p} L_{q}\left(c_{j, p}\right) V_{n, q} \widetilde{k}\left(h_{n}\left(1-c_{j}\right) c_{p}\right), \\
& n=0, \ldots, N-1 ; \quad j=1,2,
\end{aligned}
$$

where we introduced the ( $\alpha$-dependent) weights

$$
\lambda_{j, p}:=\int_{c_{j}}^{1}\left(\nu-c_{j}\right)^{-\alpha} L_{j, p}(\nu) d \nu, \quad j, p=1,2 .
$$

An analytic expression for these weights will be given in short. For the integrals in the lag term $P_{n, j}$ the analysis goes similarly. There the interpolation nodes are the same as the collocation points $c_{j}$, so

$$
V_{l}\left(x_{l}+\nu h_{l}\right) \widetilde{k}\left(x_{l}-x_{n, j}+\nu h_{l}\right) \approx \sum_{q=1}^{2} L_{q}(\nu) V_{l, q} \widetilde{k}\left(x_{l}-x_{n, j}+c_{q} h_{l}\right),
$$

which gives the product integration rule for the lag-term

$$
\hat{P}_{n, j}=\sum_{l=n+1}^{N-1} h_{l}^{1-\alpha}\left(\sum_{q=1}^{2} \omega_{l, j, q} V_{l, q} \widetilde{k}\left(x_{l}-x_{n, j}+c_{q} h_{l}\right)\right),
$$

and the weights are defined as

$$
\begin{array}{r}
\omega_{l, j, q}:=\int_{0}^{1}\left[\frac{x_{l}-x_{n, j}}{h_{l}}+\nu\right]^{-\alpha} L_{q}(\nu) d \nu, \\
j, q=1,2 ; \quad l=n+1, \ldots, N-1 .
\end{array}
$$

To conclude, the entries $f_{n, j}$ are approximated as follows:

$$
f_{n, j} \approx \hat{P}_{n, j}+\hat{A}_{n, j}, \quad n=0, \ldots, N-1, \quad j=1,2 .
$$

B.2. Weights on positive semiaxis. Formulas for the weights in (62) and (65) are derived next. We start with $\omega_{l, j, q}$ in (65). To simplify the notation let us introduce the numbers

$$
d_{l, j}=\frac{x_{l}-x_{n, j}}{h_{l}} .
$$

This amounts to saying that the following integral is to be evaluated analytically:

$$
\int_{0}^{1}\left(d_{l, j}+\nu\right)^{-\alpha} L_{q}(\nu) d \nu
$$


Recall that the Lagrange interpolation polynomials $L_{q}(\nu)$ are given by the formulas

$$
L_{1}(\nu)=\frac{\nu-c_{2}}{c_{1}-c_{2}}, \quad L_{2}(\nu)=\frac{\nu-c_{1}}{c_{2}-c_{1}} .
$$

We carry out the computation only for $L_{1}$, as for $L_{2}$ it goes similarly. Adding and subtracting $d_{l, j}$ in the expression $\nu-c_{2}$ produces two integrals that may be easily evaluated, indeed,

$$
\int_{0}^{1}\left(d_{l, j}+\nu\right)^{-\alpha}\left(\nu-c_{2}\right) d \nu=\int_{0}^{1}\left(d_{l, j}+\nu\right)^{-\alpha+1} d \nu-\left(d_{l, j}+c_{2}\right) \int_{0}^{1}\left(d_{l, j}+\nu\right)^{-\alpha} d \nu .
$$

Integrating above yields the formulas for the weights

$$
\left\{\begin{aligned}
\omega_{l, j, 1} & =\frac{\left(d_{l, j}+1\right)^{2-\alpha}-\left(d_{l, j}\right)^{2-\alpha}}{(2-\alpha)\left(c_{1}-c_{2}\right)}-\left(d_{l, j}+c_{2}\right) \frac{\left(d_{l, j}+1\right)^{1-\alpha}-\left(d_{l, j}\right)^{1-\alpha}}{(1-\alpha)\left(c_{1}-c_{2}\right)} \\
\omega_{l, j, 2} & =\frac{\left(d_{l, j}+1\right)^{2-\alpha}-\left(d_{l, j}\right)^{2-\alpha}}{(2-\alpha)\left(c_{2}-c_{1}\right)}-\left(d_{l, j}+c_{1}\right) \frac{\left(d_{l, j}+1\right)^{1-\alpha}-\left(d_{l, j}\right)^{1-\alpha}}{(1-\alpha)\left(c_{2}-c_{1}\right)}
\end{aligned}\right.
$$

To compute $\lambda_{j, p}$, let us change to the variable $\nu=c_{j}+\left(1-c_{j}\right) \rho$. This simplifies the Lagrange interpolation polynomials $L_{j, q}(\nu)$ as the following equalities show:

$$
L_{j, 1}(\nu)=\frac{\nu-c_{j, 1}}{c_{j, 2}-c_{j, 1}}=\frac{v-c_{j}-\left(1-c_{j}\right) \rho}{\left(1-c_{j}\right)\left(c_{2}-c_{1}\right)}=\frac{\rho-c_{1}}{c_{2}-c_{1}}=L_{1}(\rho) .
$$

Notice that $c_{j} \neq 1$ to simplify in this expression. Analogously one finds that $L_{j, 2}(\nu)=$ $L_{2}(\rho)$. Consequently, the expression

$$
\lambda_{j, p}=\left(1-c_{j}\right)^{1-\alpha} \int_{0}^{1} \rho^{-\alpha} L_{p}(\rho) d \rho
$$

gives an alternative way to evaluate these weights. Thus, we may use the previously derived formulas for the $\omega$-weights with $d_{l, j} \equiv 0$ to arrive at the expressions for the $\lambda$-weights:

$$
\left\{\begin{array}{l}
\lambda_{j, 1}=\frac{\left(1-c_{j}\right)^{1-\alpha}}{c_{1}-c_{2}}\left[\frac{1}{2-\alpha}-\frac{c_{2}}{1-\alpha}\right] \\
\lambda_{j, 2}=\frac{\left(1-c_{j}\right)^{1-\alpha}}{c_{2}-c_{1}}\left[\frac{1}{2-\alpha}-\frac{c_{1}}{1-\alpha}\right] .
\end{array}\right.
$$

Appendix C. Integral equation over the negative semiaxis. For the negative semiaxis the discretization is very similar to the one explained in Appendix B. We keep the same notation from the positive situation and summarize the procedure. The integral equation for the negative semiaxis reads

$$
g(x)=\int_{-\infty}^{0}(u(x+y)-u(x)) \frac{e^{-G|y|}}{|y|^{1+\alpha}} d y .
$$

This expression reduces, after integration by parts, to

$$
g(x)=-\int_{-\infty}^{0} v(x+y) \hat{k}(y) d y
$$


Here we have introduced the function

$$
\hat{k}(-w):=\int_{-\infty}^{-w} \frac{e^{-G|\zeta|}}{|\zeta|^{1+\alpha}} d \zeta, \quad w>0,
$$

and $v$ stands for the derivative of $u$ with respect to the space variable. The subscript "-" for the negative kernel is again omitted. An expression in terms of the incomplete gamma function exists, namely, $\hat{k}(-w)=G^{\alpha} \Gamma(-\alpha, G w)$. The Lipschitz property (17) is now invoked to allow the above integration by parts, along with a growth property for large negative values; i.e.,

- there exist constants $C_{3}>0$ and $0<C_{4}<G$ such that $|u(z)| \leq C_{3} e^{C_{4}|z|}$ for $z \rightarrow-\infty$.

Similarly to the situation on the positive semiaxis, one takes into account the singularity of the kernel $\hat{k}$. It is therefore convenient to introduce the kernel

$$
\widetilde{k}(w):=w^{\alpha} \hat{k}(-w), \quad w>0,
$$

and focus on the discretization of the integral equation

$$
g(x)=-\int_{-\infty}^{x}(x-z)^{-\alpha} v(z) \widetilde{k}(x-z) d z .
$$

Considering the collocation parameters

$$
0<c_{1}<c_{2} \leq 1
$$

the collocation mesh is defined as in (23). To discretize (76) write

$$
g_{n, j}=-\int_{a}^{x_{n, j}}\left(x_{n, j}-z\right)^{-\alpha} V(z) \widetilde{k}\left(x_{n, j}-z\right) d z .
$$

As for the positive semiaxis, one applies the product integration rule with a suitable choice of interpolation points. Summarizing, the quantities $g_{n, j}$ are approximated as follows:

$$
g_{n, j} \approx \hat{B}_{n, j}+\hat{Q}_{n, j}, \quad n=0, \ldots, N-1, \quad j=1,2 .
$$

The first component is computed with the formula

$$
\hat{B}_{n, j}=-h_{n}^{1-\alpha} \sum_{p=1}^{2} \sum_{q=1}^{2} \lambda_{j, p} L_{q}\left(c_{j, p}\right) V_{n, q} \widetilde{k}\left(h_{n} c_{j}\left(1-c_{p}\right)\right), \quad j=1,2,
$$

the weights $\lambda_{j, p}$ being

$$
\left\{\begin{array}{l}
\lambda_{j, 1}=\frac{\left(c_{j}\right)^{1-\alpha}}{c_{2}-c_{1}}\left[\frac{1}{2-\alpha}-\frac{1-c_{2}}{1-\alpha}\right], \\
\lambda_{j, 2}=\frac{\left(c_{j}\right)^{1-\alpha}}{c_{1}-c_{2}}\left[\frac{1}{2-\alpha}-\frac{1-c_{1}}{1-\alpha}\right],
\end{array}\right.
$$

and $c_{j, p}=c_{j} c_{p}$. The second component in this sum corresponds to an approximation of the lag-term,

$$
\hat{Q}_{n, j}=-\sum_{l=0}^{n-1} h_{l}^{1-\alpha}\left[\sum_{q=1}^{2} \omega_{l, j, q} V_{l, q} \widetilde{k}\left(x_{n, j}-x_{l}-c_{q} h_{l}\right)\right],
$$


where the weights for the negative semiaxis are

$$
\left\{\begin{array}{l}
\omega_{l, j, 1}=-\frac{\left(d_{l, j}\right)^{2-\alpha}-\left(d_{l, j}-1\right)^{2-\alpha}}{(2-\alpha)\left(c_{1}-c_{2}\right)}+\left(d_{l, j}-c_{2}\right) \frac{\left(d_{l, j}\right)^{1-\alpha}-\left(d_{l, j}-1\right)^{1-\alpha}}{(1-\alpha)\left(c_{1}-c_{2}\right)}, \\
\omega_{l, j, 2}=-\frac{\left(d_{l, j}\right)^{2-\alpha}-\left(d_{l, j}-1\right)^{2-\alpha}}{(2-\alpha)\left(c_{2}-c_{1}\right)}+\left(d_{l, j}-c_{1}\right) \frac{\left(d_{l, j}\right)^{1-\alpha}-\left(d_{l, j}-1\right)^{1-\alpha}}{(1-\alpha)\left(c_{2}-c_{1}\right)} .
\end{array}\right.
$$

As for the positive integral, a compensation constant is necessary to smooth the kernel. The constant in this situation is

$$
\theta_{-}=\frac{G^{\alpha} \Gamma(2-\alpha)}{\alpha(1-\alpha)}
$$

so that

$$
k_{-}(x):=\widetilde{k}(x)+\theta_{-} x^{\alpha},
$$

and the splitting of the integral equation with respect to the new kernel reads

$$
g(x)=-\int_{-\infty}^{x}(x-z)^{-\alpha} v(z) k_{-}(z-x) d z+\theta_{-} \int_{-\infty}^{x} v(z) d z .
$$

Appendix D. Stability analysis. In order to gain some insight into the stability of the resulting discretization of the PIDE, we perform here a Von Neumann stability analysis; however, we do this for a "model" version of the time-dependent problem under consideration.

Let $\alpha \in(0,1)$ and consider the equation

$$
u_{t}=-\int_{0}^{x}(x-z)^{-\alpha} u_{z}(z, t) d z, \quad x \in[0,1] .
$$

To simplify the analysis, we concentrate on a special case of the collocation method, namely, the grid is assumed equidistant, i.e., $h_{n}=h$, and the collocation parameters are $c_{1}=0.5$ and $c_{2}=1$. These choices give the collocation points $x_{n, 1}=(n+1 / 2) h$ and $x_{n, 2}=(n+1) h$.

Let us analyze the Euler scheme for the midpoints $x_{n, 1}$ (for the other collocation points the analysis goes similarly). By $u_{n+\frac{1}{2}}$ we denote the value of the solution at the midpoints. With the notation in Appendix C, (80), (81), (82), and (83), we may write the explicit collocation scheme as

$$
\begin{aligned}
u_{n+\frac{1}{2}}^{m}-u_{n+\frac{1}{2}}^{m-1}=-\gamma h\left[\sum _ { p = 1 } ^ { 2 } \lambda _ { 1 , p } \left(L_{1}\left(c_{1, p}\right)\right.\right. & \left.V_{n, 1}^{m-1}+L_{2}\left(c_{1, p}\right) V_{n, 2}^{m-1}\right) \\
& \left.+\sum_{l=0}^{n-1}\left(\omega_{l, 1,1} V_{l, 1}^{m-1}+\omega_{l, 1,2} V_{l, 2}^{m-1}\right)\right] .
\end{aligned}
$$

Here we have introduced the parameter

$$
\gamma:=\frac{k}{h^{\alpha}}
$$


Our objective is to show that by controlling the parameter $\gamma$ one attains stability of the Euler scheme.

For the collocation parameters under consideration the corresponding collocation derivatives in (87) are

$$
V_{n, 1}^{m-1}=\frac{u_{n+1}^{m-1}-u_{n}^{m-1}}{h}, \quad V_{n, 2}^{m-1}=\frac{3 u_{n+1}^{m-1}-4 u_{n+\frac{1}{2}}^{m-1}+u_{n}^{m-1}}{h},
$$

and the Lagrange interpolations are

$$
L_{1}\left(c_{1,1}\right)=3 / 2, \quad L_{1}\left(c_{1,2}\right)=1, \quad L_{2}\left(c_{1,1}\right)=-1 / 2, \quad L_{2}\left(c_{1,2}\right)=0 .
$$

Substituting (90) and (89) into (87) and collecting terms we arrive at the scheme

$$
u_{n+\frac{1}{2}}^{m}=u_{n+\frac{1}{2}}^{m-1}-\gamma \sum_{l=0}^{n}\left[\left(A_{l}+3 B_{l}\right) u_{l+1}^{m-1}-4 B_{l} u_{l+\frac{1}{2}}^{m-1}+\left(B_{l}-A_{l}\right) u_{l}^{m-1}\right]
$$

where $A_{n}=\frac{3}{2} \lambda_{1,1}+\lambda_{1,2}, B_{n}=-\frac{1}{2} \lambda_{1,1}, A_{l}=\omega_{l, 1,1}$, and $B_{l}=\omega_{l, 1,2}(l=0 \ldots n-1)$. The $\lambda$-weights are straightforwardly computed using (71), yielding

$$
\lambda_{1,1}=\frac{2^{\alpha}}{2-\alpha}, \quad \lambda_{1,2}=\frac{2^{\alpha}}{2-\alpha}-\frac{2^{\alpha-1}}{1-\alpha} .
$$

The $\omega$-weights are computed using (70) with

$$
d_{l, 1}=n-l+1 / 2, \quad d_{l, 2}=n-l+1 .
$$

A method to obtain a necessary condition for stability is to substitute the Fourier modes $u_{l}^{m}=g^{m} e^{i l \theta}$ into the scheme and thereafter perform the necessary simplifications. This procedure is commonly known as discrete Von Neumann stability analysis. Applied to our case it produces the so-called amplification factor

$$
g(\theta)=1-\gamma \sum_{l=0}^{n}\left[\left(A_{l}+3 B_{l}\right) e^{i(l-n+1 / 2) \theta}-4 B_{l} e^{i(l-n) \theta}+\left(B_{l}-A_{l}\right) e^{i(l-n-1 / 2) \theta}\right] .
$$

If the scheme is stable, it is also stable on a particular solution. Hence, the amplification factor must satisfy the requirement

$$
|g(\theta)| \leq 1
$$

We refer the reader to [35] for details on the amplification factor in connection with the Von Neumann stability analysis. We are looking for a condition of the form $\gamma \leq C(\alpha)$ such that (95) holds. Instead of deriving this condition for model equation (86), we present in Table 5 some sharp values of $C(\alpha)$ for distinct choices of $\alpha$, and two representative examples are displayed in Figure 5.

A limiting case is distinguished: For $\alpha \rightarrow 0$ one notices that $C(\alpha) \rightarrow 1$. This behavior is intuitively correct as for $\alpha=0$, equation (86) reduces to the ODE $u_{t}=$ $-u+u(0)$. For $\alpha=0$ the condition says $k \leq 1$, which is the necessary condition for explicit Euler, since the amplification factor for $u_{t}=-u+u(0)$ is

$$
g(\theta)=1-k+k e^{-i m \theta} .
$$


TABLE 5

Values of $C(\alpha)$ as a function of $\alpha$.

\begin{tabular}{c|c|c|c|c|c|c|c|c|c|c|c}
\hline$\alpha$ & 0.05 & 0.1 & 0.2 & 0.3 & 0.4 & 0.5 & 0.6 & 0.7 & 0.8 & 0.9 & 0.95 \\
\hline$C(\alpha)$ & 0.99 & 0.97 & 0.92 & 0.85 & 0.76 & 0.67 & 0.57 & 0.46 & 0.2 & 0.03 & 0.007 \\
\hline
\end{tabular}
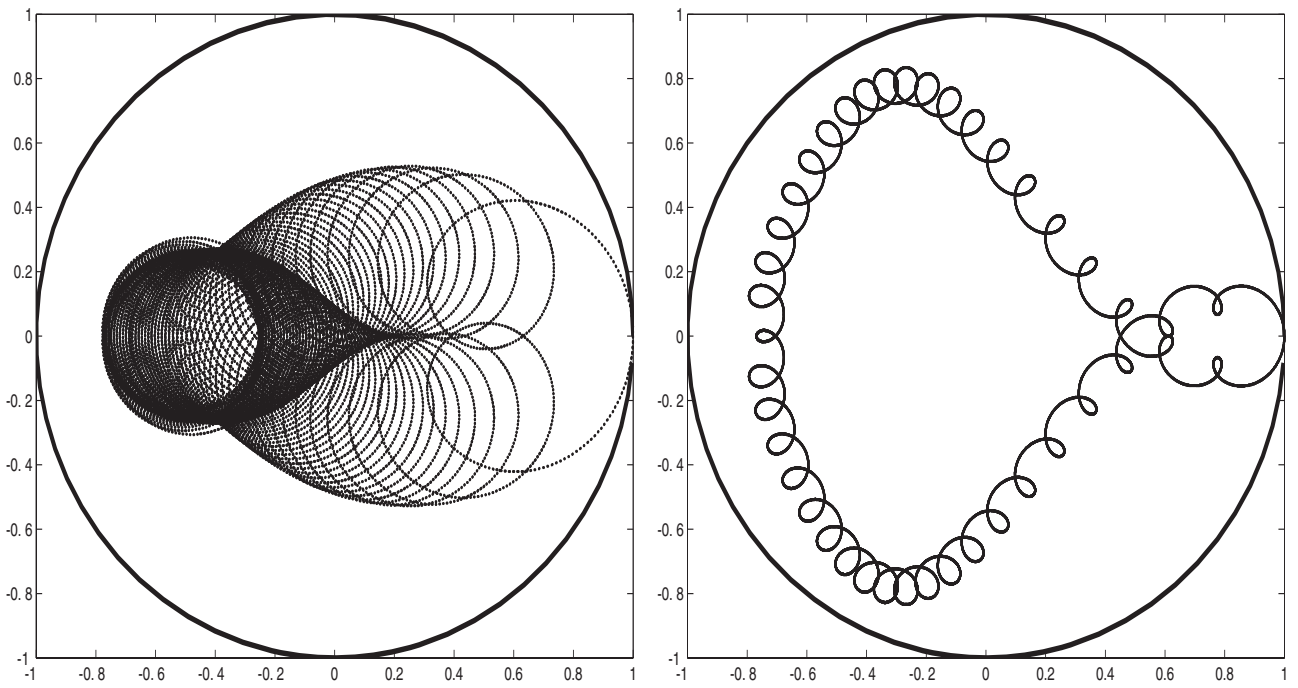

Fig. 5. Values of $g(\theta)$ inside unit circle, $k=C(\alpha) h^{\alpha}$. Left: $\alpha=0.3, N=50$. Right: $\alpha=0.7, N=20$, with $C(0.3)=0.85$ and $C(0.7)=0.46$ as in Table 5 .

Then, $|g| \leq 1$ implies $|\operatorname{Re} g| \leq 1$, which in turn implies $k \leq 1$.

The insight gained here from model equation (86) with explicit Euler, i.e., the $\alpha$ dependent constant $C(\alpha)$ so that (95) holds, is in full agreement with the observations regarding stability for our target discrete PIDE, discretized with explicit BDF2, in the numerical experiments of section 4 . We include here a numerical experiment with the test equation (86). Three values of $\alpha$ are chosen to illustrate that the values in Table 5 are sharp; see Table 6 . This table shows that

$$
k \leq C(\alpha) h^{\alpha}
$$

is in fact a necessary condition for stability. Namely, if (96) is not satisfied, the scheme is not stable. It should be mentioned that $C(\alpha)$ depends weakly on $N$, and the values in Table 5 are conservative in the sense that they are found with $N=20$. For larger $N$, the pictures in Figure 5, for example, tend to shrink.

It can be observed in Table 6 that the number of time steps needed for stability grows with increasing $\alpha$. For $\alpha \leq 0.7$ the number of time steps remains reasonable, and explicit time-stepping can efficiently be used. For $\alpha$ closer to 1, however, one may need to consider an implicit time discretization. For $\alpha=0.9$ we see a difference of 200 time steps for a consistency between the stability analysis and the numerical experiment. The reason for this large difference lies in the assumption that a satisfactory stability criterion is $|g| \leq 1$ (95). In fact, a weaker stability criterion that is both necessary and sufficient is given by $|g| \leq 1+\tilde{C} k$, for $\tilde{C}$ independent of $h$ and $k$. This typically gives larger circles, so $C(\alpha)$ in the table represents a conservative estimate. The tendency with respect to stability is, however, clear. 
TABLE 6

Stability experiments on test equation (86) with $T=1$. The symbol " $v$ " means "holds" and " $X$ " means "does not hold." The initial condition is taken as $u(x, 0)=\exp (x)$.

\begin{tabular}{c|c|c|c|c}
\hline$\alpha$ & $N$ & $N_{t}$ & Stability & Equation (96) \\
\hline 0.05 & 100 & 2 & $\mathrm{v}$ & $\mathrm{v}$ \\
\hline \hline & 100 & 6 & $\mathrm{v}$ & $\mathrm{V}$ \\
0.3 & 100 & 4 & $\mathrm{X}$ & $\mathrm{X}$ \\
\hline \hline & 100 & 30 & $\mathrm{v}$ & $\mathrm{v}$ \\
0.5 & 100 & 10 & $\mathrm{X}$ & $\mathrm{X}$ \\
\hline \hline & 100 & 140 & $\mathrm{v}$ & $\mathrm{V}$ \\
0.7 & 100 & 80 & $\mathrm{X}$ & $\mathrm{X}$ \\
& 100 & 40 & $\mathrm{X}$ & $\mathrm{X}$ \\
\hline \hline \multirow{2}{*}{0.9} & 20 & 400 & $\mathrm{v}$ & $\mathrm{V}$ \\
& 20 & 300 & $\mathrm{X}$ & $\mathrm{X}$ \\
& 20 & 200 & $\mathrm{X}$ & \\
\hline
\end{tabular}

Acknowledgment. We would like to thank the referees for valuable comments.

\section{REFERENCES}

[1] M. Abramowitz and I. A. Stegun, eds., Handbook of Mathematical Functions with Formulas, Graphs, and Mathematical Tables, Dover, New York, 1992. Reprint of the 1972 edition.

[2] L. Alili And A. E. Kyprianou, Some remarks on first passage of Lévy processes, the American put and pasting principles, Ann. Appl. Probab., 15 (2005), pp. 2062-2080.

[3] A. Almendral, Numerical valuation of American options under the CGMY process, in Exotic Option Pricing and Advanced Lévy Models, W. Schoutens, A. Kyprianou, and P. Wilmott, eds., Wiley, Chichester, UK, 2005.

[4] A. Almendral and C. W. Oosterlee, On American Options under the Variance Gamma Process, Tech. report, Delft University of Technology, Delft, The Netherlands, 2004.

[5] A. Almendral and C. W. Oosterlee, Numerical valuation of options with jumps in the underlying, Appl. Numer. Math., 53 (2005), pp. 1-18.

[6] L. Andersen and J. Andreasen, Jump-diffusion processes: Volatility smile fitting and numerical methods for option pricing, Review of Derivatives Res., 4 (2000), pp. 231-262.

[7] O. E. BARndorfF, Processes of normal inverse Gaussian type, Finance Stoch., 2 (1998), pp. 4168.

[8] F. Black And M. S. Scholes, The pricing of options and corporate liabilities, J. Political Economy, 7 (1973), pp. 637-54.

[9] S. I. Boyarchenko and S. Z. Levendorskĭ, Non-Gaussian Merton-Black-Scholes Theory, Adv. Ser. Stat. Sci. Appl. Probab. 9, World Scientific, River Edge, NJ, 2002.

[10] H. BRunner, Collocation Methods for Volterra Integral and Related Functional Differential Equations, Cambridge Monogr. Appl. Comput. Math. 15, Cambridge University Press, Cambridge, UK, 2004.

[11] H. Brunner and P. J. van der Houwen, The Numerical Solution of Volterra Equations, CWI Monogr. 3, North-Holland, Amsterdam, 1986.

[12] J. Y. Campbell, A. W. Lo, And A. C. MacKinlay, The Econometrics of Financial Markets, Princeton University Press, Princeton, NJ, 1997.

[13] P. P. Carr, H. Geman, D. B. Madan, and M. Yor, The fine structure of asset returns: An empirical investigation, J. Business, 75 (2002), pp. 305-332.

[14] P. P. Carr and D. B. Madan, Option valuation using the Fast Fourier Transform, J. Comp. Finance, 2 (1999), pp. 61-73.

[15] P. P. Carr, D. B. Madan, And E. C. Chang, The variance Gamma process and option pricing, European Finance Review, 2 (1998), pp. 79-105.

[16] R. Cont And P. Tankov, Financial Modelling with Jump Processes, Chapman \& Hall, Boca Raton, FL, 2004.

[17] J. C. Cox AND S. A. Ross, The valuation of options for alternative stochastic processes, J. Financial Economics, 3 (1976), pp. 145-166.

[18] S. R. DAS AND S. Foresi, Exact solutions for bond and option prices with systematic jump risk, Review of Derivatives Res., 1 (1996), pp. 7-24. 
[19] Y. D'Halluin, P. A. Forsyth, and K. R. Vetzal, Robust numerical methods for contingent claims under jump diffusion processes, IMA J. Numer. Anal., 43 (2005), pp. 1596-1626.

[20] B. Dupire, Pricing with a smile, RISK Magazine, 1 (1999), pp. 18-20.

[21] E. EBerlein, Application of generalized hyperbolic Lévy motions to finance, in Lévy Processes, Birkhäuser Boston, Boston, 2001, pp. 319-336.

[22] P. EgGermont, Stability and robustness of collocation methods for Abel-type integral equations, Numer. Math., 45 (1984), pp. 431-445.

[23] E. Hairer, C. Lubich, AND M. SChlichte, Fast numerical solution of weakly singular Volterra integral equations, J. Comput. Appl. Math., 23 (1988), pp. 87-98.

[24] D. Heath And M. Schweizer, Martingales versus PDEs in finance: An equivalence result with examples, J. Appl. Probab., 37 (2000), pp. 947-957.

[25] S. Heston, A closed-form solution for options with stochastic volatility with applications to bond and currency options, Rev. Financ. Stud., 6 (1993), pp. 327-343.

[26] D. J. Higham, An Introduction to Financial Option Valuation, Cambridge University Press, Cambridge, UK, 2004.

[27] A. Hirsa And D. B. Madan, Pricing American options under variance Gamma, J. Comp. Finance, 7 (2004), pp. 63-80.

[28] J. Hull AND A. White, The pricing of options with stochastic volatilities, J. Finance, 42 (1987), pp. 281-300.

[29] S. G. Kou, A jump diffusion model for option pricing, Management Science, 48 (2002), pp. 1086-1101.

[30] Y. K. Kwok, Mathematical Models of Financial Derivatives, 2nd ed., Springer Finance, Springer-Verlag, Singapore, 1998.

[31] P. Linz, Analytical and Numerical Methods for Volterra Equations, SIAM Stud. Appl. Math. 7, SIAM, Philadelphia, 1985.

[32] A. M. Matache, P. A. Nitsche, and C. Schwab, Wavelet Galerkin pricing of American options on Lévy driven assets, Quant. Finance, 5 (2005), pp. 403-424.

[33] R. C. Merton, Option pricing when the underlying stocks are discontinuous, J. Financ. Econ., 5 (1976), pp. 125-144.

[34] W. Schoutens, Lévy Processes in Finance: Pricing Financial Derivatives, John Wiley \& Sons, Chichester, UK, 2003.

[35] J. C. Strikwerda, Finite Difference Schemes and Partial Differential Equations, The Wadsworth \& Brooks/Cole Mathematics Series, Wadsworth \& Brooks/Cole Advanced Books \& Software, Pacific Grove, CA, 1989.

[36] C. VAn LoAn, Computational Frameworks for the Fast Fourier Transform, Frontiers Appl. Math. 10, SIAM, Philadelphia, 1992.

[37] P. Wilmott, J. Dewynne, and S. Howison, Option Pricing, Oxford Financial Press, Oxford, UK, 1993.

[38] S. Zhang And J. Jin, Computation of Special Functions, John Wiley \& Sons, New York, 1996. 